\title{
Nonequilibrium Steady State in a Quantum System: One-dimensional Transverse Ising Model with Energy Current
}

\author{
Tibor Antal and Zoltán Rácz \\ Institute for Theoretical Physics, Eötvös University \\ 1088 Budapest, Puskin u. 5-7, Hungary \\ László Sasvári \\ Department of Solid State Physics, Eötvös University \\ 1088 Budapest, Múzeum krt. 6-8, Hungary
}

\begin{abstract}
We study the nonequilibrium steady states of an Ising chain in a transverse field, $h$, by investigating the effect of a field, $\lambda$, which drives the current of energy. The zero-temperature, $h-\lambda$ phase diagram is determined exactly and it is found that the energy current appears continuously above a threshold, $\lambda>\lambda_{c}(h)$. The long-range magnetic order (present for $h<h_{c}, \lambda<\lambda_{c}$ ) is destroyed in the current-carrying state where the correlations are characterized by power-law, oscillatory decay. The mechanism which generates the power-law correlations in the current-carrying state is discussed.
\end{abstract}

PACS numbers: 05.50.+q, 05.70.Ln, 75.10.Jm

Nonequilibrium steady states have been much investigated but a general theory comparable to the theory of equilibrium systems is still lacking. A traditional approach to nonequilibrum phenomena starts with the construction of deterministic phenomenological equations, followed by a linear or nonlinear stability analysis of the solutions and ends up with the study of the effect of fluctuations by adding noise terms to the equations [1.2. An alternative and somewhat more microscopic approach is the introduction of kinetic Ising models where the nonequilibrium steady states are produced by the spins being driven by external fields or by being in contact with several heat baths at different temperatures [3, : 1 .

Both of these approaches have lead to successful descriptions of a number of particular phenomena but they have not been very helpful in deducing general conclusions. The main problem is that the observed properties of nonequilibrium steady states are strongly dependent on the details of the dynamics (even the presence or absence of a phase transition or the type of the phase transitions, etc. may depend on the details of the transition probabilities). From a theoretical point of view, the problem is further complicated by the absence of the detailed balance condition which provides at least some constraints on the possible forms of dynamics of nearequilibrium fluctuations.

A way to avoid the above problem of too much freedom is to investigate systems which, in contrast to models such as the Ising model, have natural (quantum mechanical) dynamics. A nontrivial model with intrinsic dynamics investigated in this paper is the one-dimensional $(d=1)$ transverse Ising model. Once a quantum mechanical model is chosen, the next question is how to make it a nonequilibrium system. A possible route is to couple it to heat baths just as in the case of the various kinetic Ising models. Unfortunately, the coupling of quantum mechanical systems to classical heat baths is not quite understood and leads again to much arbitrariness. In order to avoid this, we follow a different path by noting that nonequilibrium steady states are always associated with some kind of current (of heat, particle, momentum, etc.). Thus we make a nonequilibrium steady state in a quantum system by imposing a current on the system. For example, in case of the transverse Ising model where the only conserved quantity is the energy, we shall constrain the system to have an energy current. It is hard to treat, however, a 'microcanonical' type constraint of fixed energy flow and so, we shall actually use the easier, 'canonical' description which consists of introducing a field which drives the given (energy) current.

The next question is what to investigate. An obvious choice is to study the changes produced by the presence of a current in the orderings and in the phase diagram of the system. In particular, for the $d=1$ transverse Ising model, one can investigate whether the $T=0$ Ising transition remained in the same universality class and whether new phases emerged as a consequence of the energy current. Furthermore, it is known that currents in nonequilibrium steady states usually generate long-range correlations [5]. Thus it is natural to inquire if similar phenomena would occur in a quantum nonequilibrium steady state.

The practical realization of the above program consists of the following steps:

i) The starting point is the Hamiltonian which, for the $d=1$ transverse Ising model, has the form:

$$
\hat{H}_{I}=-\sum_{\ell} \sigma_{\ell}^{x} \sigma_{\ell+1}^{x}-\frac{h}{2} \sum_{\ell} \sigma_{\ell}^{z}
$$


where the spins are represented by Pauli spin matrices $\sigma_{\ell}^{\alpha}(\alpha=x, y, z)$ at sites $\ell=1,2, \ldots, N$ of a $d=1$ periodic chain $\left(\sigma_{N+1}^{\alpha}=\sigma_{1}^{\alpha}\right)$, and $h$ is the transverse field in units of the Ising coupling.

ii) The local energy current, $\hat{J}_{\ell}$, is calculated by taking a time derivative of the energy density, using the quantum mechanical equation of motion, and representing the result as a divergence of the energy current $(\hbar=1$ is used):

$$
\hat{J}_{\ell}=\frac{h}{4} \sigma_{\ell}^{y}\left(\sigma_{\ell-1}^{x}-\sigma_{\ell+1}^{x}\right)
$$

iii) The 'macroscopic' current $\hat{J}=\sum_{\ell} \hat{J}_{\ell}$ is added to $\hat{H}_{I}$ with a Lagrange multiplier, $-\lambda$,

$$
\hat{H}=\hat{H}_{I}-\lambda \hat{J}
$$

Note that the energy current, $\hat{J}$, is associated with $\hat{H}_{I}$ and not with the new Hamiltonian, $\hat{H}$. We also emphasize that $\hat{H}$ is just another equilibrium Hamiltonian, it differs from $\hat{H}_{I}$ by an extra term which breaks the leftright symmetry of $\hat{H}_{I}$. Finding the ground state of $\hat{H}$, however, gives us the minimum energy state of $\hat{H}_{I}$ which carries an energy current, $J=\langle\hat{J} / N\rangle$ (brackets \langle\rangle denote the expectation value in the ground-state of $\hat{H}$ ). Thus the ground-state properties of $\hat{H}$ provide us with the properties of the nonequilibrium steady states of the transverse Ising model.

iv) The correlations and ordering properties of the system are investigated as a function of $\lambda$. Of course, one can also find that value of $\lambda$ which generates a given energy current, $J=\langle\hat{J} / N\rangle$, in the transverse Ising model and then all steady state properties can be obtained in terms of $J$.

It should be noted that the terms in $\hat{J}$ can be rearranged so that the energy current becomes a current through the bonds:

$$
\hat{J}=\sum_{\ell} \hat{\bar{J}}_{\ell}=\frac{h}{4} \sum_{\ell}\left(\sigma_{\ell}^{x} \sigma_{\ell+1}^{y}-\sigma_{\ell}^{y} \sigma_{\ell+1}^{x}\right)
$$

This form is remarkable because $\hat{\bar{J}}_{\ell}$ can be recognized as the Dzyaloshinskii-Moriya interaction in the theory of weak ferromagnetism [6]. Moreover, the current of the $z$ component of the magnetization in the transverse XY model is also of the above form [7], and similar terms (apart from an overall factor of $i$ which makes the Hamiltonian nonhermitian) appear in the description of driven diffusive systems [8], as well as in the Hamiltonian description of the direct electric field of the 6 -vertex model [9].

We turn now to the solution of the problem. Since $\left[\hat{H}_{I}, \hat{J}\right]=0$, the Hamiltonian $\hat{H}$ is diagonalized using the same transformations which diagonalize $\hat{H}_{I}$ [10,11: first, creation-annihilation operators are introduced $\left(a_{\ell}^{ \pm}=\right.$ $\left.\sigma_{\ell}^{x} \pm i \sigma_{\ell}^{y}\right)$, then the Jordan-Wigner transformation 10 is used to transform them into fermion operators $\left(c_{\ell}, c_{\ell}^{+}\right)$ and, finally, a Bogoljubov transformation [12] is employed on the $q$ and $-q$ components of the Fourier transforms of $c_{\ell}$-s. As a result one finds the spectrum of excitation energies as $\omega_{q}=\left|\Lambda_{q}\right|$ where

$$
\Lambda_{q}=\frac{1}{2}\left(\sqrt{1+h^{2}+2 h \cos q}+\zeta \sin q\right) .
$$

Here the wave numbers are restricted to $-\pi \leq q \leq \pi$ in the thermodynamic limit $(N \rightarrow \infty)$ and we introduced $\zeta=\lambda h / 2$ which appears to be the natural variable instead of $\lambda$. Fig.1 displays the spectrum for $h=0.5$ and various $\zeta$ and one can see that the $q \rightarrow-q$ symmetry of the spectrum is broken for $\zeta \neq 0$. For small $\zeta$ when $\Lambda_{q} \geq 0$, however, the ground-state remains that of the transverse Ising model $(\zeta=0)$ and, accordingly, no energy current flows $(J=0)$. This rigidity of the ground state against the symmetry-breaking field which drives the energy current is a consequence of the facts that the fermionic spectrum of the transverse Ising model has a gap and that the operator $\hat{J}$ commutes with $\hat{H}_{I}$. Actually, the rigidity persists even at the critical point $h_{c}=1$ where the gap disappears. The reason for this is more subtle and related to the fact that the critical spectrum of $\hat{H}_{I}$ has a finite slope at $q= \pm \pi$.

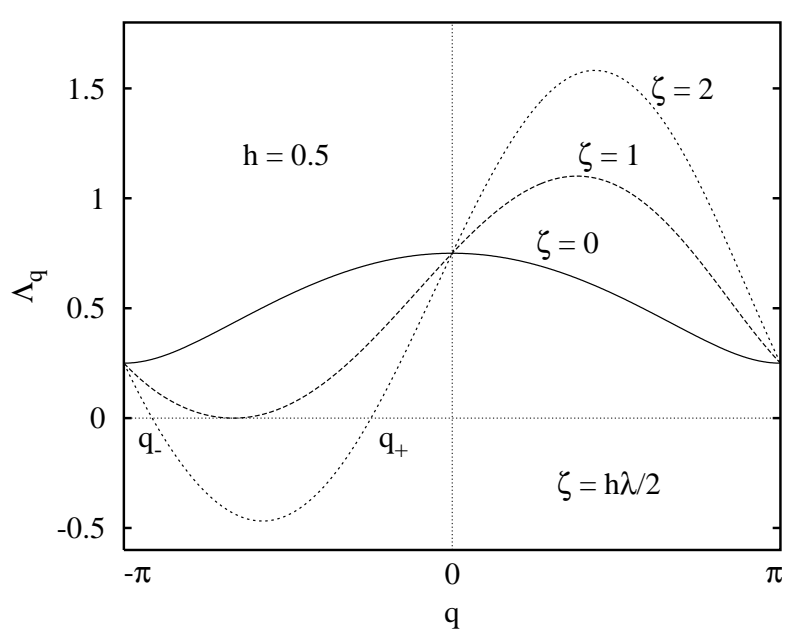

FIG. 1. Spectrum of the transverse Ising model in the presence of a field $(\lambda)$ which drives the current of energy. The excitation energies are given as $\omega_{q}=\left|\Lambda_{q}\right|$. The qualitative picture is the same at all transverse fields $h$ except at $h=1$ where the $q= \pm \pi$ gap disappears.

The ground-state properties do change when $\Lambda_{q}<0$ in an interval $\left[q_{-}, q_{+}\right]$since these $q$ states become occupied and $q_{ \pm}$depends on both $h$ and $\zeta$. The line $\zeta_{c}(h)$ which borders the region of unchanged transverse Ising behavior is obtained from the conditions

$$
\Lambda_{q}=0 \quad, \quad \partial \Lambda_{q} / \partial q=0
$$

and the solution 


$$
\zeta_{c}=\frac{\lambda_{c} h}{2}= \begin{cases}\zeta_{c}^{+}=h & h \geq 1 \\ \zeta_{c}^{-}=1 & h<1\end{cases}
$$

is displayed on the phase diagram (Fig.2) as a solid line. Fig.2 also shows the phase boundary (dashed line) between the magnetically ordered $(h<1, \zeta<1)$ and disordered $(h \geq 1, \zeta<h)$ transverse Ising regions. Since the ground state is independent of $\zeta$ for $\zeta<\zeta_{c}$, the transition across the dashed line is a second order transition belonging to the $d=2$ Ising universality class [11].

In the following we shall argue that the $\zeta>\zeta_{c}$ region can be considered as a distinct phase since i) the energy current is nonzero, ii) there is no long-range magnetic order, and iii) the magnetic correlations are oscillatory with amplitudes decaying as a power of distance.

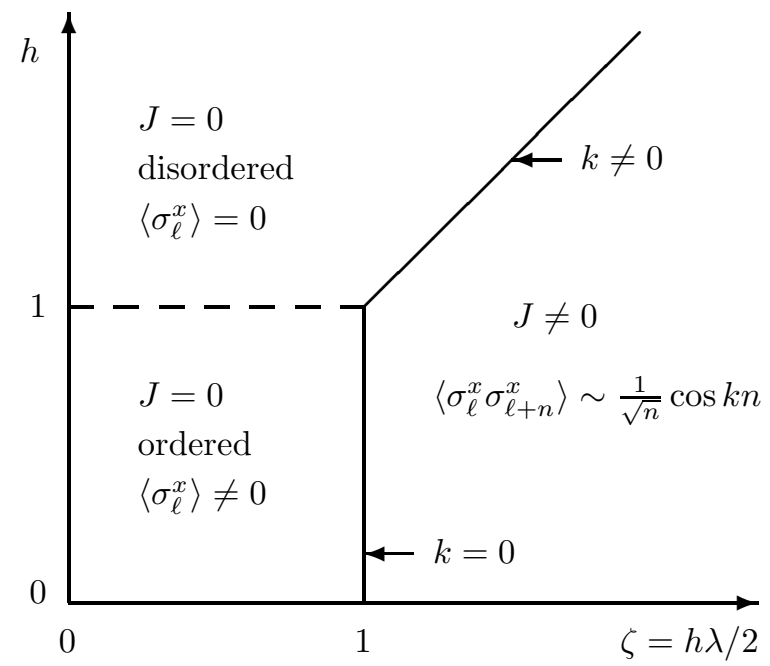

FIG. 2. Phase diagram in the $h-\zeta$ plane where $h$ is the transverse field while $\zeta=h \lambda / 2$ is the effective field which drives the current of energy. Power-law correlations are present in the current-carrying phase $(J \neq 0)$ and on the Ising critical line (dashed line).

The energy current is expressed as a product of two fermion operators and can be calculated exactly. It is zero for $\zeta \leq \zeta_{c}$ while, for $\zeta>\zeta_{c}$, one obtains:

$$
J=\langle\hat{J} / N\rangle=\frac{h}{4 \pi \zeta^{2}} \sqrt{\left(\zeta^{2}-h^{2}\right)\left(\zeta^{2}-1\right)} .
$$

At the phase boundary, $\zeta_{c}$, we have $J \rightarrow 0$ as

$$
J \sim \begin{cases}\left(\zeta-\zeta_{c}\right)^{1 / 2} & h \neq 1 \\ \zeta-\zeta_{c} & h=1 .\end{cases}
$$

Thus the $\zeta_{c}$ line can be considered as a line of second order transition provided $J$ is viewed as an order parameter.

There are several other quantities which change nonanalytically as we cross into the current carrying phase.
For example, the change in the $z$ component of the magnetization at fixed $h$ is given by

$$
\left\langle\sigma_{\ell}^{z}\right\rangle_{\zeta}-\left\langle\sigma_{\ell}^{z}\right\rangle_{\zeta_{c}} \sim\left\{\begin{array}{cc}
\left(\zeta-\zeta_{c}\right)^{1 / 2} & h>1 \\
\zeta-\zeta_{c} & h=1 \\
\left(\zeta-\zeta_{c}\right)^{3 / 2} & h<1 .
\end{array}\right.
$$

The different exponents obtained for $h>1$ and $h<1$ reflect the fact that the $J \neq 0$ phase neighbors magnetically ordered $\left(\left\langle\sigma_{\ell}^{x}\right\rangle \neq 0\right)$ and magnetically disordered $\left(\left\langle\sigma_{\ell}^{x}\right\rangle=0\right)$ phases along the $\zeta_{c}^{+}$and $\zeta_{c}^{-}$portions of the $\zeta_{c}$ phase boundary.

The long-range magnetic order $\left(\left\langle\sigma_{\ell}^{x}\right\rangle \neq 0\right)$ disappears for $\zeta>\zeta_{c}$. This can be seen by investigating the $\left\langle\sigma_{\ell}^{x} \sigma_{\ell+n}^{x}\right\rangle$ correlations which can be expressed through Pfaffians [13,14. In the presence of long-range order, one should have $\left\langle\sigma_{\ell}^{x} \sigma_{\ell+n}^{x}\right\rangle \rightarrow\left\langle\sigma_{\ell}^{x}\right\rangle^{2} \neq 0$ for $n \rightarrow \infty$. Instead, we find that the correlations decay to zero at large distances as

$$
\left\langle\sigma_{\ell}^{x} \sigma_{\ell+n}^{x}\right\rangle \sim \frac{Q(h, \zeta)}{\sqrt{n}} \cos (k n)
$$

where the wavenumber, $k$, depends only on $\zeta$,

$$
k=\arccos \zeta^{-1} .
$$

The above results $(11,12)$ are exact in the $\zeta \rightarrow \infty$ limit where $Q(h, \infty)=e^{1 / 2} 2^{-4 / 3} A^{-6} \approx 0.147$ is the amplitude of the $\left\langle\sigma_{\ell}^{x} \sigma_{\ell+n}^{x}\right\rangle$ correlations of the $d=1 X Y$ model 14 ( $A \approx 1.282$ is Glaisher's constant). The connection to the $X Y$ model and the details of the calculation will be published separately [15].

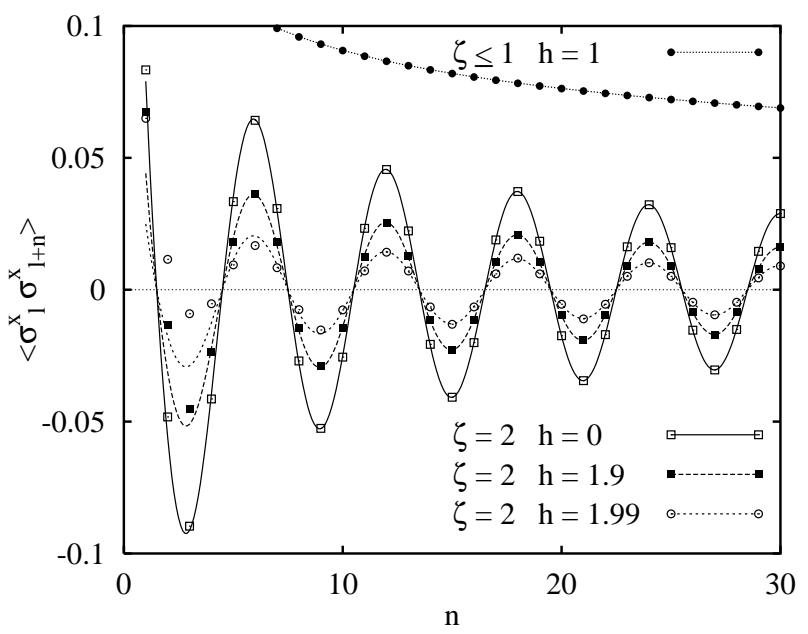

FIG. 3. The correlation function $\left\langle\sigma_{\ell}^{x} \sigma_{\ell+n}^{x}\right\rangle$ at various transverse fields $h$ with $\zeta=h \lambda / 2$ fixed at $\zeta=2$. The lines are fits to the large $n$ behavior which is assumed to be of the form $Q n^{-1 / 2} \cos k n$. Changes in the amplitude $Q$ occur mainly near the $\zeta_{c}^{+}$phase boundary $(h \rightarrow 2)$ where $Q \rightarrow 0$. Note that the convergence to the asymptotic behavior becomes slower as $h \rightarrow 2$. For comparison, we have also plotted the correlations $\left\langle\sigma_{\ell}^{x} \sigma_{\ell+n}^{x}\right\rangle \sim n^{-1 / 4}$ on the Ising critical line (dashed line on Fig.2). 
For $\zeta_{c}<\zeta<\infty$, the expressions (11, 12) are obtained from the numerical analysis of $\left\langle\sigma_{\ell}^{x} \sigma_{\ell+n}^{x}\right\rangle$ for finite $n \leq$ 100. Typical results are shown on Fig. 3. The numerical results are behaved well enough so that, in addition to the $n$ dependence of $\left\langle\sigma_{\ell}^{x} \sigma_{\ell+n}^{x}\right\rangle$, the functional form of the amplitude $Q(h, \zeta)$ can also be investigated. We find that the following expression fits the results everywhere $\left(\zeta>\zeta_{c}\right)$ except close to the phase boundaries, $\zeta_{c}^{ \pm}$, or near the $h=1$ line:

$$
Q(h, \zeta) \approx Q(h, \infty)\left(\frac{\zeta^{2}-h^{2}}{\zeta^{2}-1}\right)^{1 / 4}
$$

Near either $\zeta=\zeta_{c}^{ \pm}$or $h=1$, the convergence to the asymptotic form (11) is slow and the numerics becomes unreliable. The convergence becomes fast again on the $h=1$ line itself where the following form appears to be valid:

$$
Q(1, \zeta) \approx Q(h, \infty)\left(\frac{\zeta^{2}}{\zeta^{2}-1}\right)^{1 / 8}
$$

Power-law decay of correlations for $\zeta>\zeta_{c}$ is present in other physical quantities as well. For example, the envelopes of both $\left\langle\sigma_{\ell}^{z} \sigma_{\ell+n}^{z}\right\rangle$ and $\left\langle\hat{J}_{\ell} \hat{J}_{\ell+n}\right\rangle$ decay in the large $n$ limit as $n^{-2}$ 15]. Thus we find power-law correlations in the current carrying state in agreement with the notion that power-law correlations are a ubiquitous feature of nonequilibrium steady states [16].

Power-law correlations in quantum models are usually associated with a gapless excitation spectrum. Thus, provided the transverse Ising model can be considered as an instructive example, we can see a general connection between the emergence of power-law correlations and the presence of a current. Indeed, let us assume that a system with Hamiltonian $\hat{H}_{0}$ has a spectrum with a gap between the ground-state and the lowest excited state. Furthermore, let $\hat{J}$ be a 'macroscopic' current of a conserved quantity such that $\left[\hat{H}_{0}, \hat{J}\right]=0$. Generally, there is no current in the the ground state and adding $-\lambda \hat{J}$ to $\hat{H}_{0}$ does not change the $\langle\hat{J}\rangle=0$ result for small $\lambda$. Current can flow only if some excited states mix with the ground state and, consequently, a branch of the excitation spectrum must come down and intersect the ground-state energy in order to have $\langle\hat{J}\rangle \neq 0$. Once this happens, however, the gap disappears and one can expect power-law correlations in the current-carrying state.

Admittedly, the above argument is not strict and is just a reformulation (in general terms) of the transverse Ising results. We believe, however, that the above picture is robust and suggestive enough to warrant further studies in this direction.

In summary, we have seen that nonequilibrium steady states in quantum systems can be constructed by switching on fields which drive the current of conserved quantities. Studies of these states may be of importance since their construction is free from the arbitraryness inherent in classical nonequilibrium dynamics. On the particular example of the transverse Ising model, we could observe that there are power-law correlations in the currentcarrying states and we argued that this may be a general feature of quantum systems.

\section{ACKNOWLEDGMENTS}

We thank Z. Horváth, L. Palla, P. Ruján, G.M. Schütz, R. Stinchcombe, G. Szabó, and T. Tél for helpful discussions. This work was supported by the Hungarian Academy of Sciences Grant OTKA T 019451, and by EC Network Grant ERB CHRX-CT92-0063.

[1] M.C. Cross and P.C. Hohenberg, Rev.Mod.Phys.65, 851 (1994).

[2] B. Schmittmann and R. K. P. Zia, in Phase Transitions and Critical Phenomena, Eds. C. Domb and J.L. Lebowitz (Academic, New York, 1995).

[3] S. Katz, J. L. Lebowitz, and H. Spohn, J.Stat.Phys. 34, 497 (1984); A. DeMasi, P.A. Ferrari, and J.L. Lebowitz, Phys.Rev.Lett. 55, 1947 (1985);

[4] T. L. Hill, J.Chem.Phys.76, 1122 (1982); P. L. Garrido, A. Labarta, and J. Marro, J.Stat.Phys.49, 551 (1987).

[5] Section III in 2] and references therein.

[6] I. E. Dzyaloshinskii, J.Phys.Chem.Solids 4, 241 (1958); T. Moriya, Phys.Rev.120, 91 (1960).

[7] A study of the transverse XY model in the presence of a field which drives the current of the $z$-component of the magnetization will be published separately. T. Antal, Z. Rácz, and G.M. Schütz, unpublished.

[8] L.-H. Gwa and H. Spohn, Phys.Rev.Lett. 68, 725 (1995); M.D. Grynberg and R.B. Stinchcombe, Phys.Rev.Lett. 74, 1242 (1995). Master equations of kinetic Ising models can often be mapped onto simple quantum spin chains i.e. quantum systems without any constraint of current may be equivalent to classical nonequilibrium systems. See e.g. E. Siggia Phys.Rev. B16, 2319 (1977); G.M. Schütz, J.Stat.Phys. 79, 243 (1995) and references therein.

[9] M.P.M. den Nijs, Phys.Rev. B23, 6111 (1981).

[10] E. Lieb, T. Schultz, and D. Mattis, Ann.Phys. (N.Y.) 16, 407 (1961).

[11] P. Pfeuty, Ann.Phys. (N.Y.) 57, 79 (1970).

[12] N.N. Bogoliubov, Nuovo Cimento 7, 794 (1958).

[13] E.R. Cainello and S. Fubini, Nuovo Cimento 9, 1218 (1952).

[14] E. Baruch and B. McCoy, Phys.Rev.A3, 786 (1971).

[15] T. Antal, Z. Rácz, and L. Sasvári, unpublished.

[16] Just as in equilibrium systems, the correlations decay exponentially in the $\zeta<\zeta_{c}$ states (except on the critical line, $h=h_{c}$ ). This is not surprising since no steady-state current (local or global) flows in those states and thus they should be considered as equilibrium states. 